\title{
Myocardial Dysfunction after Esophageal Surgery
}

\section{Manuel Ruiz-Bailén ${ }^{1,2 *}$ and Jesús Cobo-Molinos}

${ }^{1}$ Department of Health Sciences, University of Jaén, Hospital Complex of Jaén, Spain

${ }^{2}$ Department of Intensive Medicine, Hospital Complex of Jaén, Spain

\begin{abstract}
Objective: Evaluate the presence of acute myocardial dysfunction after esophageal surgery.

Methodology: All esophageal surgery patients admitted to intensive care unit (ICU) from January 2008 to December 2014 were included. The follow-up was up to the third month. We were included a control group with healthy people. Cardiac stress cardiomyopathy complicating Esophagectomy were evaluated by bedside echocardiography. Also off-line examinations were realized by a hybrid speckle tracking.

Results: 48 patients were included with $64.65 \pm 13.22$ years old; $30(62.5 \%)$ were male. Mean ICU length of stay was $32.27 \pm 18.33$ days and ICU mortality was $31.25 \%$. Cumulative mortality during follow-up was $41.67 \%$. Mechanical ventilation time was $21.39 \pm 14.29$ days; cTnl peak was $1.98 \pm 0.85 \mathrm{ng} / \mathrm{mL}$ BNP peak was $378.33 \pm$ $103.01 \mathrm{pg} / \mathrm{\mu gm}$ mean peak QTc was $474.72 \pm 104.25 \mathrm{~ms}$. APACHE 2 were $21.53 \pm 11.88$ points. Strain and train rate showed segmental and global contractility alterations in all patients. However these changes were only detected in 21 patients using 2D echocardiography. The findings observed by 2-3D echocardiography were normalized in the first week but strain and strain rate were normalized during the third month.
\end{abstract}

Conclusion: A stress cardiomyopathy may occur after esophageal surgery.

Keywords: Esophageal surgery; Myocardial dysfunction; Echocardiography; Takotsubo syndrome; Stress cardiomyopathy

\section{Abbreviations}

ICU: Intensive Care Unit; BNP: Brain Natriuretic Peptide; S: Strain; SR: Strain Rate; LVEF: Left Ventricular Ejection Fraction; RVEF: Right Ventricular Ejection Fraction; VTI: Velocity Time Integral; SIRS: Systemic Inflammatory Response Syndrome; PCWP: Pulmonary Capillary Wedge Pressure

\section{Introduction}

In intensive care medicine is accepted the possible existence of a reversible myocardial dysfunction or stress cardiomyopathy in critically ill patients especially by sepsis $[1,2]$ and after cardiac arrest [3]. Although the etiology of this complication is unknown it could be circulating catecholamines or even systemic inflammatory response syndrome (SIRS). Esophageal carcinoma is strongly linked to inflammation both its genesis and evolution. The sustained inflammatory state perpetuation is considered a driver of esophageal and lung cancer and may even warn of its complications such as sutures leakage or even their prognosis [4]. Thoracic esophageal surgery releases a large number of cytokines and can become to SIRS or stress cardiomyopathy. Cardiovascular complications are frequent in esophageal postoperative having reported arrhythmias acute coronary syndrome and even two cases of Takotsubo cardiomyopathy [5-7]. Speckle tracking echocardiography may be more sensitive in detecting stress cardiomyopathy during postoperative esophageal. The aim of this study is to evaluate the presence of myocardial dysfunction after esophageal surgery during their ICU admission.

\section{Methodology}

\section{Study design}

Observational prospective study approved and supported by PAIDI CTS 606 Andalusian Health Service Project No. PI-0585-2012. It has also been approved by local and Andalusian ethics committees. Cohorts are generated from esophageal surgery patients admitted to ICU from September 2008 to December 2014. This study is limited to Intensive Care Unit of University Jaén Hospital. The follow-up study was maintained for three months.

\section{Inclusion criteria}

Esophagectomy carcinoma shock patients complicated during the first 72 hours who required mechanical ventilation and noradrenaline were included in this study. Postoperative management was the usual and established by intensivists and surgeons. Presence of an intensivist with advanced knowledge in echocardiography on admission and during the ICU length of stay.

\section{Exclusion criteria}

1) Do not accept the inclusion in the study 2) Existence of acute coronary syndrome 3) Previous history of cardiovascular disease 4) Sepsis; and 5) Patients managed without echocardiography or with poor sound quality obtained. Clinical and demographic details were studied. All echocardiographic parameters recommended by the American Society of Echocardiography were evaluated.

Included patients were given a standard transthoracic echocardiogram with SC2000 or Sequoia 512 Siemens ${ }^{\oplus}$ USA equipment. Transthoracic examination were carried in supine position; he was also conducted an off-line analysis using the software " $2 \mathrm{D}$ Image Speckle tracking hybrid Velocity Vector Image Syngo-Software Siemens US 2006-2013". Apical four-chamber orientation was used for the acquisition of left ventricular functional data. $4 \mathrm{~V} 1 \mathrm{c}$ and $4 \mathrm{Z} 1 \mathrm{c}$ probes were used. The frame rate were as high as possible $(70-120 \mathrm{f} / \mathrm{s})$ with multiple focal point. All images were optimized with gain compression and dynamic range images.

Usual echocardiographic parameters were evaluated; left ventricular

*Corresponding author: Manuel Ruiz-Bailén, Department of Health Sciences, University of Jaén, Hospital Complex of Jaén, Jaén 23071, Spain, Tel 34953212196; Fax: 34953212943; E-mail: ruizbailen@gmail.com

Received October13, 2016; Accepted November 14, 2016; Published November 22, 2016

Citation: Ruiz-Bailén M, Cobo-Molinos J (2016) Myocardial Dysfunction after Esophageal Surgery. Surgery Curr Res 6: 280. doi:10.4172/2161-1076.1000280

Copyright: ( 2016 Ruiz-Bailén M, et al. This is an open-access article distributed under the terms of the Creative Commons Attribution License, which permits unrestricted use, distribution, and reproduction in any medium, provided the original author and source are credited. 
systolic ejection fraction (LVEF) quantification by $2 \mathrm{D}$ volume and $\mathrm{E} / \mathrm{E}^{\prime}$ ratio quantification estimating the PCWP and parameters derived from speckle tracking such as strain, strain rate, longitudinal displacement and radial velocities in the left ventricle. We adopted a six segments model to assess regional and global left ventricular performance of longitudinal fibers. The region of interest was manually traced along the endomyocardial border in the six segments. Demographic and clinical data, APACHE 2 to admission, noradrenaline urinary, interlekine 6, BNP peak, troponine I values and QTc were evaluated. In addition were studied interleukin 6 plasma levels and urinary norepinephrine levels on ICU admission.

\section{Statistical analysis}

A study for quantitative variables was performed using ANOVA. Their results are presented using mean and standard deviations. Qualitative variables were presented as absolute and relative frequencies. It was performed a correlation analysis, using the Spearman test, between values of APACHE 2 levels, interleukin-6, troponin, BNP, QTc and norepinephrine administered with Strain and the Strain Rate longitudinal left ventricular.

We have used correlations between variables by Pearson's $r$ coefficient for intra-observer variability. It was regarded a $p$ value $<0.05$ as statistically significant.

\section{Results}

48 patients were included. Patients were not included in this study Transesophageal echocardiography was performed through the colonic plasty suspecting acute endocarditis, being confirmed by one. Their mean age were $64.65 \pm 13.22$ years old; $30(62.5 \%)$ were male. $21(43.75 \%)$ patients were hypertensive, $11(22.91 \%)$ patients were diabetic, 10 (20.83\%) were obese; 4 (8.34\%) were patients with Chronic Obstructive Pulmonary Disease, 16 (13.33\%) were drinkers and 24 (50\%) were smokers. Mean ICU and hospital length of stay were $32.27 \pm 18.33$ and $48.37 \pm 33.66$ days respectively. Mechanical ventilation time was 21.39 \pm 14.29 days; cTnI peak was $1.98 \pm 0.85 \mathrm{ng} / \mathrm{mL}$ BNP peak was $378.33 \pm$ $103.01 \mathrm{pg} / \mu \mathrm{g}$ mean peak QTc was $474.72 \pm 104.25 \mathrm{~ms}$. APACHE 2 were $21.53 \pm 11.88$ points. Mean maximum norepinephrine dose was $1.44 \pm$ $0.93 \mathrm{microg} / \mathrm{kg} / \mathrm{min}$. ICU mortality was $31.25 \%$. Mean norepinephrine urinary was $157.87 \pm 21.88 \mathrm{microg} / 24 \mathrm{~h}$. Mean interleukine 6 plasmatic was $57.33 \pm 11.72 \mathrm{pg} / 24 \mathrm{~h}$. Cumulative mortality during follow-up in the next three months was $41.67 \%$.

There were no significant changes in left ventricular ejection fraction. Strain and Strain rate showed segmental and global contractility alterations in all patients. However these changes were only detected in 21 patients using $2 \mathrm{D}$ echocardiography. The findings observed by $2 \mathrm{D}$ echocardiography were normalized in the first week but strain and strain rate were normalized during the third month.

4 patients had ST-segment elevation in left precordial leads simulating an acute coronary syndrome suggestive affecting the right epicardial coronary artery during first 24 hours of esophagectomy. Segmental contractility disorders in the basal segments of the left ventricle and the inferiors segments of the right ventricle with severe systolic dysfunction were detected in Echocardiography and ventriculography [LVEF $0.33 \pm 0.18$ ). Left ventricular strain (S) was $-12 \pm(2.13)$ and right shortening fractional area was $0.34 \pm 0.28$. Right ventricular S was $-17 \pm$ (5.41)]. Surprisingly the estimated left ventricular end-diastolic pressures were not very high (E/E' ratio $9.89 \pm$ 5.12). These ECG changes were normalized within 72 hours.

Of the 48 patients studied, 29 had segmental alterations in contractility viewable by $2 \mathrm{D}$; however in all of them there was a decrease of segmental strain in septoapical and apicolateral segments; except in 4 patients with ST segment elevation maintained where changes in the basal segments were detected. The findings observed by $2 \mathrm{D}$ echocardiography are normalized in the first week but the strain was normalized during the third month.

Correlations made by Spearman test, only detected association between Left ventricular longitudinal Strain with Levels of urinary norepinephrine ( $\mathrm{R} 2=0.547, \mathrm{n}=27$ patients, $\mathrm{p}=0.001$ ) (Figures 1 and 2 ).

\section{Discussion}

Critically ill patients myocardial dysfunction has been described in other situations especially during sepsis $[1,2]$ but could have a common etiology SIRS [2-4]. They have been described cases reports with ST segment disorders with normal epicardial coronary arteries. In the present study has been observed in 4 patients the same dynamic changes of ST segment with normal epicardial coronary arteries and increase enzymatic all compatible with critically ill patients' myocardial dysfunction or stress cardiomyopathy.

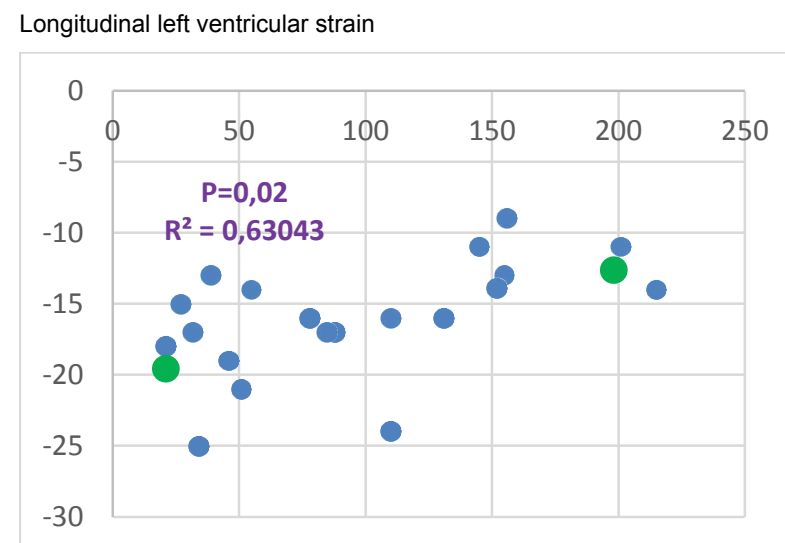

\section{Norepinephrine peak}

Figure 1: Correlation between peak values of urinary norepinephrine and left ventricular longitudinal strain values.

Longitudinal left ventricular strain

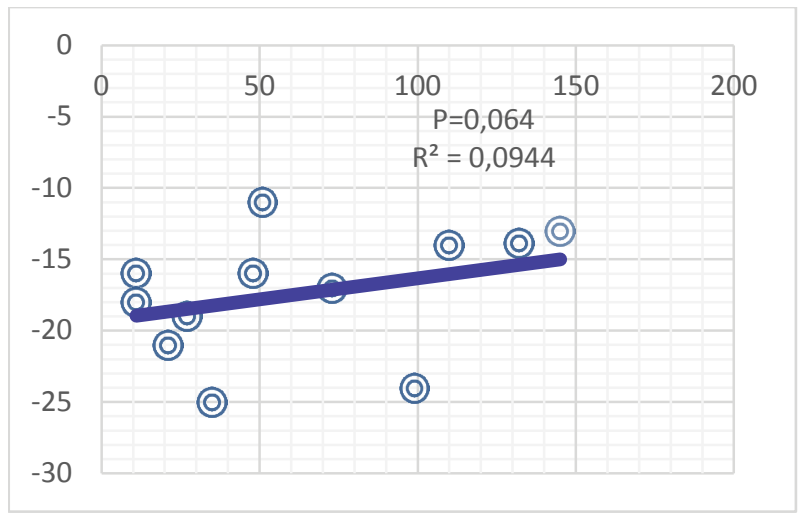

Urinary interleukine 6 peak

Figure 2: Correlation between peak values of interleukine 6 and left ventricular longitudinal strain values. 


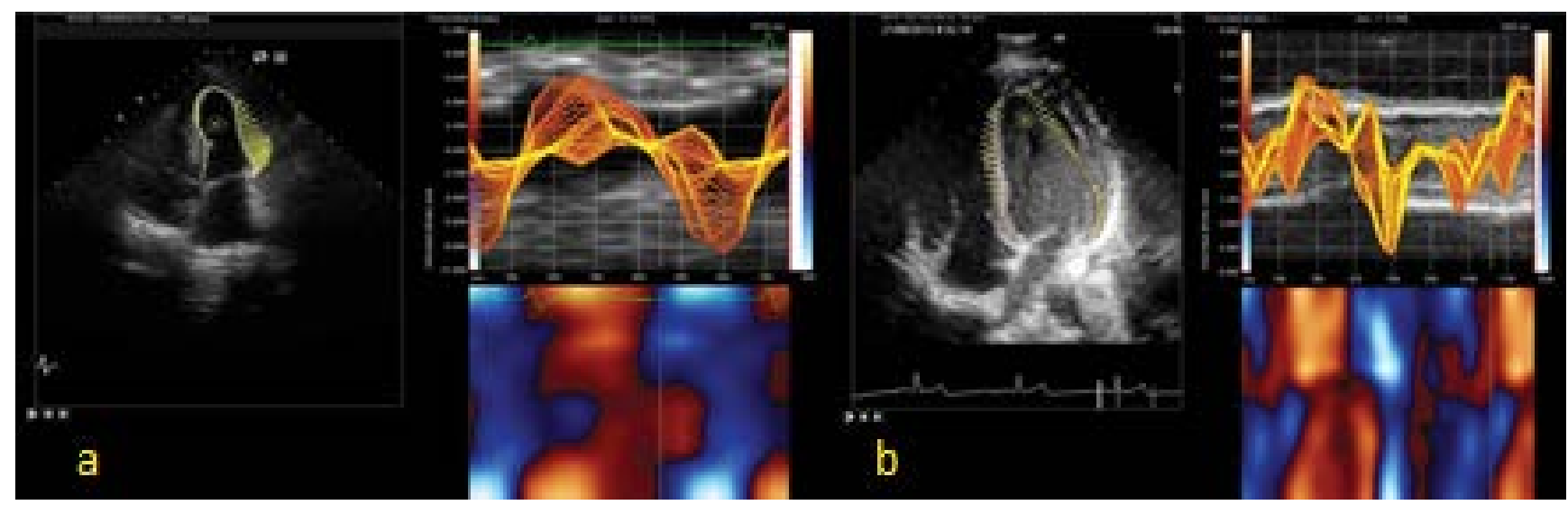

Figure 3: a) The figure shows the systolic and diastolic abnormalities in acute perioperative. b) The figure shows normal myocardial performance at 3 months follow-up.

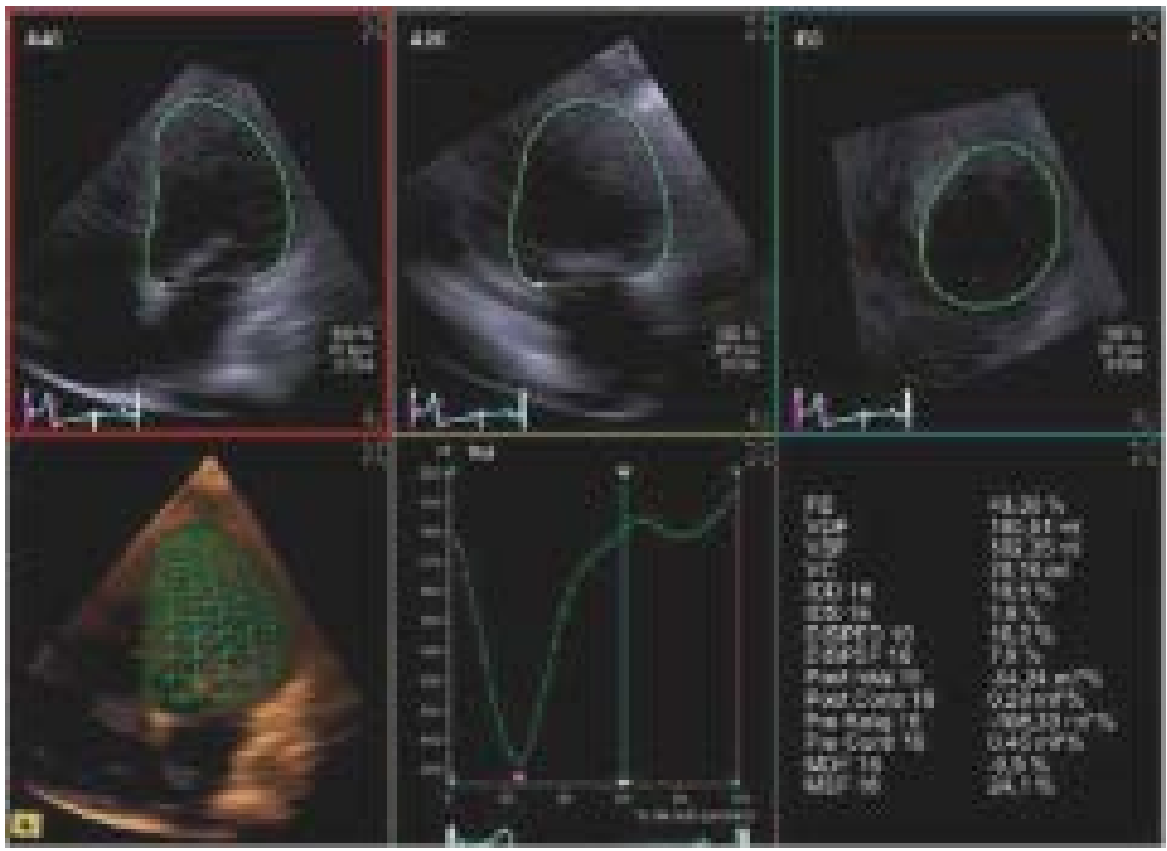

Figure 4: In some patients there has been severe left ventricular dilation recoverable after the event 3D echocardiography.

The most interesting finding of this manuscript is that ventricular ejection fractions were not modified by volumetric studies, but whether there was an unexpected rise in PCWP. This supports the findings in ventricular strain, suggesting that these patients suffer a deterioration of cardiac performance. Myocardial dysfunction in these patients is likely to produce a systolic and diastolic myocardial dysfunction. This effect is logical if we understand that myocardial performance is a continuum. We also observed by speckle tracking decrease in all patients S SR and systolic longitudinal velocities peaks suggestive of myocardial dysfunction after the esophagectomy. In the study was segmental contractility disorders in the apical segments especially septoapical and lateroapical and a basal involvement; similar to takotsubo or reverse takotsubo cardiomyopathy respectively.

Esophageal surgery produces a large release of cytokines and catecholamines that may be related to surgical aggression or even own malignity. It is therefore logical that respiratory and renal injury may perhaps generate a potentially reversible myocardial dysfunction that could worse patient prognosis. Interestingly, our work detects association on left ventricular strain with urinary norepinephrine. However we did not have sufficient statistical power to detect a significant correlation between the longitudinal strain with interleukin 6 . This effect which could be supporting the mechanism of inflammation and stress. Another interesting finding is that most patients show increase QTc probably explained by the alterations in membranes of cardiomyocytes.

The findings observed by 2D/3D echocardiography are normalized in the first week but the strain was normalized during the third month (Figures 3 and 4). These findings could assume that the Speckle Tracking could be a useful tool in intensive care medicine to be more sensitive. The design of this study can make us understand that there are limitations. However we believe that this design is suitable for the main objective. One limitation of this study is probably the existence of a selection bias, as patients who were included were extremely critically 
Citation: Ruiz-Bailén M, Cobo-Molinos J (2016) Myocardial Dysfunction after Esophageal Surgery. Surgery Curr Res 6: 280. doi:10.4172/21611076.1000280

ill patients with very high APACHE score. This study does not allow ass's mortality in esophagectomy patients or its complications; but it is useful for detecting myocardial dysfunction after esophagectomy.

\section{Conclusion}

Critically ill patients with esophagectomy may be complicated during early postoperative by a myocardial dysfunction that could be detected by speckle tracking echocardiography.

\section{References}

1. Ruiz Bailén M (2002) Reversible myocardial dysfunction in critically ill, noncardiac patients: a review. Crit Care Med 30: 1280-1290.

2. Romero-Bermejo FJ, Ruiz-Bailen M, Gil-Cebrian J, Huertos-Ranchal MJ (2011) Sepsis-induced cardiomyopathy. Curr Cardiol Rev 7: 163-183.
3. Ruiz-Bailén M, Aguayo de Hoyos E, Ruiz-Navarro S, Díaz-Castellanos MA Rucabado- Aguilar L, et al. (2005) Reversible myocardial dysfunction after cardiopulmonary resuscitation. Resuscitation 66: 175-181.

4. Niwa Y, Koike M, Iwata N, Kobayashi D, Tanaka C, et al. (2014) Effect of landiolol hydrochloride on tachyarrhythmia after esophagectomy. Hepatogastroenterology 61: 1546- 1555

5. Bosch DJ, Muijs CT, Mul VE, Beukema JC, Hospers GA, et al. (2014) Impact of neoadjuvantchemoradiotherapy on postoperative course after curative-inten transthoracic esophagectomy in esophageal cancer patients. Ann Surg Onco 21: 605-611.

6. Gangadhar TC, Von der Lohe E, Sawada SG, Helft PR (2008) Takotsubo cardiomyopathy in a patient with esophageal cancer: a case report. J Med Case Rep 2: 379.

7. Schweizer MT, Mehta R, Salgia R, Villaflor VM (2011) Takotsubo cardiomyopathy in a patient with squamous cell esophageal carcinoma. J Clin Oncol 29: 598-600. 